\title{
Effects of Diversity Combining Techniques and Carrier Frequency offset on Enhancement of BER Mobile Network
}

\author{
Ibrahim Khider \\ Sudan University of Science \\ and Technology
}

\author{
Adam Mohamed \\ Sudan University of Science \\ and Technology
}

\author{
Nidaa Elsheikh \\ Sudan University of Science \\ and Technology
}

\begin{abstract}
Fading is a fundamental problem in wireless communication. However Space time block code scheme is suggested to overcome this problem. In the paper various combination of transmit and receive antenna was considered, a mathematical model was driven and applied using simulation. The relation between signal to noise ratio and bit error rate was plotted. From results it is noted that the receiver diversity better than transmitter diversity, but if both diversity is used that gives better results as shown in Multiple input Multiple Output (MIMO), also signal to noise ratio (SNR) and bit error rate (BER) with carrier frequency offset (CFO) and without carrier frequency offset was compared.
\end{abstract}

\section{Keywords}

Fading, Diversity combining technique, Bit Error Rate, Space time block code scheme, MIMO, carrier frequency offset, Simulation

\section{INTRODUCTION}

In a world of increasing mobility, there is a growing need for people to communicate with each other and have timely access to information regardless of the location of the individuals or the information. A phone call placed from a commuter train may close a business deal, remote access to medical records by a paramedic may save a life, or a request for reconnaissance updates by a soldier with a handheld device may affect the outcome of a battle. Each of these instances of mobile communications poses an engineering challenge that can be met only with an efficient, reliable, wireless communication network [1]. However Fading can cause poor performance in a communication system because it can result in a loss of signal power without reducing the power of the noise. This signal loss can be over some or all of the signal bandwidth. Fading can also be a problem as it changes over time: communication systems are often designed to adapt to such impairments, but the fading can change faster than the adaptations can be made [2].

If one signal path undergoes a deep fade at a particular point of time, another independent path may have a strong signal. By having more than one path to select from, both the instantaneous and average SNR can be improved in the receiver by a large amount which is the fundamental idea of diversity techniques.

There are various types of diversity used in communication systems operating over fading channels [3]. They are: Space
Diversity, Frequency Diversity, Time Diversity, Polarization Diversity, and Multipath Diversity.

Space-Time Codes (STCs) have been implemented in cellular communications as well as in wireless local area networks. Space time coding is performed in both spatial and temporal domain introducing redundancy between signals transmitted from various antennas at various time periods. It can achieve transmit diversity and antenna gain over spatially un-coded systems without sacrificing bandwidth. The research on STC focuses on improving the system performance by employing extra transmits antennas. In general, the designs of STC amounts to finding transmit matrices that satisfy certain optimality criteria. Constructing STC, researchers have to trade-off between three goals: simple decoding, minimizing the error probability, and maximizing the information rate. The essential question is: How can we maximize the transmitted date rate using a simple coding and decoding algorithm at the same time as the bit error probability is minimized [4][5][7].

\section{Mathematical Models}

Space time block code scheme is suggested by Mr. Siavash M Alamouti is simple transmit diversity technique in wireless communication, in normal if we have source of sequence $\left\{S_{1}, S_{2}, S_{3}, \ldots \ldots \ldots S_{n}\right\}$ we send in the first time slot $\mathrm{S} 1$ and second time slot send $\mathrm{S} 2$ and the nth time slot send $\mathrm{S}_{\mathrm{n}}$ and so on . Alamouti suggest that grouped the symbols of two groups and send first time slot send S1 and S2 from first and second antenna and send in second time slot $\mathrm{S}_{1}{ }^{*}$ and $\mathrm{S}_{2}{ }^{*}$ from first and second antenna and so on. Still we need two time slots to send two symbols. We consider various combination of transmit and receive antenna and then drive the mathematical model to apply MATLAB simulation.

Firstly we considering transmit diversity Multiple Input Single Output (MISO).

- Two transmit antenna and one receive antenna Fist time slot, the receiver single

$$
\begin{aligned}
& y_{1}=h_{1} x_{1}+h_{2} x_{2}+n_{1} \\
& =\left[\begin{array}{ll}
h_{1} & h_{2}
\end{array}\right]\left[\begin{array}{l}
\mathrm{x}_{1} \\
\mathrm{x}_{2}
\end{array}\right]+\mathrm{n}_{1} \\
& 2^{\text {nd }} \text { Time slot, the receiver single } \\
& y_{2}=-h_{1} \mathrm{x}_{2}^{8}+\mathrm{h}_{2} \mathrm{x}_{1}^{8}+\mathrm{n}_{2}
\end{aligned}
$$




$$
=\left[\begin{array}{ll}
h_{1} & h_{2}
\end{array}\right]\left[\begin{array}{c}
-\mathrm{x}_{2}^{8} \\
\mathrm{x}_{1}^{8}
\end{array}\right]+\mathrm{n}_{2}
$$

$\varepsilon\left(S^{H} \mathrm{~S}\right)$

$$
\leq\left[\left(\left|h_{1}^{2}\right|+\left|h_{2}^{2}\right|\right)\left(N a+2 \varepsilon\left(\left|x_{k}(1)\right|^{2}\right)\right]\right.
$$

$\mathrm{y}_{1}, \mathrm{y}_{2}$ received symbol/ signal at $\mathrm{S}_{1} \& \mathrm{~S}_{2}$ and $\mathrm{X}_{1}, \mathrm{X}_{2}$ transmitted symbol respectively. $\mathrm{h}_{1}, \mathrm{~h}_{2}$ fading channel coefficient $n_{1}, n_{2}$ AWGN of $S_{1}$ and $S_{2}$ with variance $\sigma_{n}{ }^{2}=$ $\mathrm{N}_{\mathrm{o}} / 2$ per real dimension

So the received signal at two times instants, namely $r_{1}$ and $r_{2}$ is given by:

$$
\left[\begin{array}{l}
\gamma_{1} \\
\gamma_{2}
\end{array}\right]=\left[\begin{array}{cc}
h_{1} & h_{2} \\
h_{2}^{*} & -h_{1}^{*}
\end{array}\right]+\left[\begin{array}{l}
x_{1} \\
x_{2}^{*}
\end{array}\right]+\left[\begin{array}{l}
n_{1} \\
n_{2}^{8}
\end{array}\right]
$$

The received signal after maximum ratio combining (MRC) is given by:

$$
\begin{aligned}
& {\left[\begin{array}{l}
y_{1} \\
y_{2}
\end{array}\right]=\left[\begin{array}{cc}
h_{1}^{8} & h_{2} \\
h_{2}^{8} & -h_{1}
\end{array}\right]+\left[\begin{array}{l}
f_{1} \\
\gamma_{2}^{8}
\end{array}\right]} \\
& =\left[\begin{array}{cc}
\left|h_{1}^{2}\right|+\left|h_{2}^{2}\right| & 0 \\
0 & \left|h_{1}^{2}\right|+\left|h_{2}^{2}\right|
\end{array}\right]\left[\begin{array}{l}
\mathrm{x}_{1} \\
\mathrm{x}_{2}
\end{array}\right]+ \\
& {\left[\begin{array}{l}
h_{1}^{*} n_{1}+h_{2} n_{2}^{8} \\
h_{2}^{*} n_{1}+h_{1} n_{2}^{*}
\end{array}\right]} \\
& \mathrm{SNR}=\frac{\left(\left|h_{1}^{2}\right|+\left|h_{2}^{2}\right|\right)^{2} \frac{k_{S}}{z}}{g\left(\left(h_{1}^{*} n_{1}+h_{2} n_{2}^{*}\right)\left(h_{2}^{*} n_{1}+h_{1} n_{2}^{*}\right)^{*}\right)} \\
& =\left|h_{1}^{2}\right|+\left|h_{2}^{2}\right| \frac{E_{S}}{2 N s}
\end{aligned}
$$

$E_{s}$ is energy for each transmitted symbol.

The received signal after $K_{t h}$ MS with perfect knowledge of channel at transmitter is given by [4][5][6]

$$
r_{K}(\mathrm{t})=H_{k} \sum_{i=1}^{K} w_{i} b_{i}(t)+n_{K}(\mathrm{t})
$$

$W_{k}$ Is selected to be orthogonal to the erroneous channel matrix $\quad H_{K}=H_{K}+E_{K}$

Thus received signal is given by

$r_{k}(\mathrm{t})=\widetilde{H}_{K} b_{k}(t)+\widetilde{n}_{k}(\mathrm{t})$

Where $\tilde{n}_{k}$ (t) is AWGN and co-channel interference (CCI) from other users due to imperfect cancelation.

$x_{k z}(\mathrm{t})=E_{K} \sum_{i=1, i=k}^{k} w_{i} b_{i}(\mathrm{t})$

The noise component $n_{K}$ is replaced by the combination of noise AWGN \&CCI

$$
\begin{aligned}
\mathrm{S} & =h_{1}^{8} \tilde{n}_{k g}(1)+h_{2}^{8} \tilde{n}_{k g}(2)^{\mathrm{H}} \\
& =h_{1}^{8} n_{k}(1)+h_{2} n_{k}(2)^{\mathrm{H}}+h_{1}^{8} x_{k}(1)+h_{2} x_{k}(2)^{\mathrm{H}} .
\end{aligned}
$$

By approximation of $x_{k}(2)$ by $x_{k}(1)$, the covariance of the equivalent noise component is:
Refer to equation (3) SNR above, the SNR after STBC decoding is:

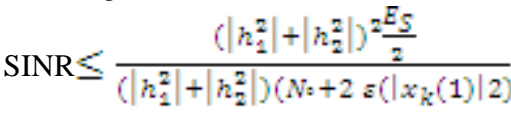

$$
\begin{aligned}
& =\frac{\mid h_{1}^{2}\|+\| h_{x}^{2} \|}{1+2 a\left(\left\|x_{k}(1)\right\| 2\right) f_{W s}} \times \frac{E_{S}}{2 N s}
\end{aligned}
$$

For simplicity refer to equation (6)

$$
\varepsilon\left(\left|x_{k}(1)\right|^{2}\right)=(\mathrm{K}-1) \sigma_{M S E}^{2} E_{S} \text {. . . }
$$

Substituting (10) into (9) we obtain the final result of SNR and system estimation error at transmitter is:

$\operatorname{SINR} \leq \frac{\left|h_{1}^{2}\right|+\mid h_{2}^{2} \|}{1+2(K-1) \sigma_{\text {WISE }}^{E_{S}} f_{N o}} \times \frac{E_{S}}{2 N w}$

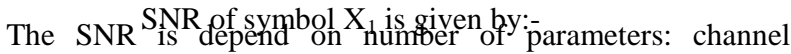
estimation error, signal power level $E_{S} / \mathrm{N}_{\mathrm{o}}$

If only one user $\mathrm{K}=1$ or channel is perfect $\left(\sigma_{\mathrm{MSE}}^{2}=0\right)$ diversity gain of two is obtained.

The relation of BER with average SNR is:

$P_{e}=\left[\frac{1}{2}(1-\mu)\right]^{2} \sum_{K=0}^{1} \frac{(K+1) x_{x}}{K_{x}^{v}}\left[\frac{1}{2}(1+\mu)\right]^{K}$

Where $\mu=\sqrt{\frac{\bar{y}}{1+\bar{y}}}$

Average SINR

$\bar{Y}=\frac{E_{S} g_{2 N o}}{1+2(K-1) \sigma_{\text {MISE }} E_{S}}$

Equation (12) is final derivation of transmitter diversity with 2Tx and $1 R x$

Note: there is different between $\mathrm{k}$ and ( $\mathrm{K}$ is number of users). For 4Tx and $1 \mathrm{Rx}$ is similarly the relation become:

$P_{e}=\left[\frac{1}{2}(1-\mu)\right]^{4} \sum_{K=0}^{3} \frac{(K+3)}{K ! 3 \pm}\left[\frac{1}{2}(1+\mu)\right]^{\mathrm{K}}$

$\bar{Y}=\frac{E_{S}{ }_{4 N,}}{1+4(K-1) \sigma_{\text {MSE }}^{n} E_{S}}$

General form of $\mathrm{n}$ TX and $1 \mathrm{Rx}$ :

$\frac{E_{S f_{\mathrm{neWs}}}}{1+\mathrm{n}(K-1) \sigma_{\mathrm{NSE}}^{\mathrm{n}} E_{S / N \mathrm{~W}}}$

- Secondly the receiver diversity

Same as transmit diversity but different at equation of average SNR as shown

One TX and two Rx 


$$
\begin{aligned}
& P_{e}=\left[\frac{1}{2}(1-\mu)\right]^{4} \sum_{K=0}^{3} \frac{(K+3)^{\pi}}{K_{x}^{ \pm} 3_{x}^{*}}\left[\frac{1}{2}(1+\mu)\right]^{\mathrm{K}} . \\
& \text { Where } \mu=\sqrt{\frac{\bar{y}}{1+\bar{y}}} \\
& \bar{Y}=\frac{E_{S_{W}}}{1+2(K-1) \sigma_{\text {WISE }} E_{S}}
\end{aligned}
$$

One TX and four Rx:

$$
\bar{Y}=\frac{E_{S} g_{W}}{1+4(K-1) \sigma_{M S E}^{n} E_{S}}
$$

General form of $1 \mathrm{TX}$ and $\mathrm{n} \mathrm{RX}$

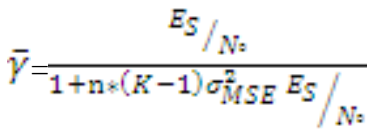

Thirdly multiple input multiple output( MIMO)

In MIMO is combination between different numbers of transmit and receive antenna, the final derivation of average SNR is:

-Two TX and two Rx is:

$\bar{\gamma}=\frac{E_{S_{2 N}}}{1+4(K-1) \sigma_{\text {MISE }} E_{S}} \times 4 \sigma_{\alpha}^{2}$

General form of $\mathrm{n}$ TX and $\mathrm{m} \mathrm{RX:}$

$\bar{\gamma}=\frac{E_{S} f_{n \times N o}}{1+n \times m e(K-1) \sigma_{D S E}^{n} E_{S}} \times \mathrm{n} * \mathrm{~m} * \sigma_{\alpha}^{2}$

- Fourthly Carrier Frequency Offset takes account.

In wireless communication each transmitter use the carrier frequency to transmit and the receiver perform demodulation to recover the signal by generating the same carrier using local oscillator, however is not perfectly generate same frequency to match TX frequency, because carrier shift due to Doppler effect ,this is referred to as frequency offset .

To optimize the performance the CFO must minimize as possible the derivation as:

$$
\begin{aligned}
& f=\frac{1}{\sqrt{2 \pi \sigma_{g}^{2}}} \exp \left(-\frac{g^{2}}{2 \sigma_{g}^{2}}\right) \\
& \text { Where } \sigma_{\varepsilon}^{2} \text { variance of } \varepsilon \text { and } \varepsilon \text { is normalized CFO of the } \\
& \text { channel } \\
& \text { The average SNR of } 2 \mathrm{Tx} \text { and } 2 \mathrm{Rx} \text { is given by: } \\
& \bar{Y}=\frac{E_{S} f_{4 N B}}{1+\left[4(K-1) \sigma_{\text {MISE }}^{n} E_{S} N\right]_{8 f}} \times 4 \sigma_{\alpha}^{2}
\end{aligned}
$$

\section{Simulation Description}

We applied the simulation in mobile network environment using matlab, the work is done by driving the diversity models for both transmitter and receiver. Then the signal to noise ratio is chosen to be variable from $0 \mathrm{~dB}$ to $25 \mathrm{~dB}$; according to the mathematical model. The relation between SNR and BER was found. Also the number of users $(\mathrm{K})$ is considered for variety cases (one at first case and two at the second case). By choosing the different number of transmitter (Tx) and receiver (Rx) as $(1,2,4, \ldots \ldots, \mathrm{n}) \mathrm{Tx}$ and $(1,2,4, \ldots \ldots . \mathrm{m}) \mathrm{Rx}$, these number of Tx and Rx give the schemes of MISO,SIMO,SISO and MIMO. Finally applied the model of carrier frequency offset due to doppler effect and the results with and without CFO are compared

\section{Results and Discussion}

Figure 1 represents transmitter diversity and receiver diversity, two TX and one Rx, as shown the Rx diversity is better than TX diversity which in TX diversity the SNR at 25 $\mathrm{dB}$ the BER is greater than $10^{-5}$. At $0 \mathrm{~dB}$ the BER is greater than $10^{-1}$ compared to Rx diversity with two Rx and one TX the SNR at $25 \mathrm{~dB}$ the BER is less than $10^{-5}$ and at $0 \mathrm{~dB}$ the BER is less than $10^{-1}$, this indicate that the Rx diversity is better than TX diversity. On the other hand if the number of users is increased as shown in figure 2, however the SNR is increased after certain value the BER is not changed whatever the value of SNR. Figure 3: shows that as we increased the number of TX and Rx, we achieve better BER with same SNR. Figure 4: shows the MIMO with CFO and without CFO, the BER is affected by the CFO, if it's not considered in account the BER is better than presence of CFO.In figure 5: MIMO for two users, in this case the curve of BER with SNR for MIMO with CFO is not affected by number of users as in figure 2. 


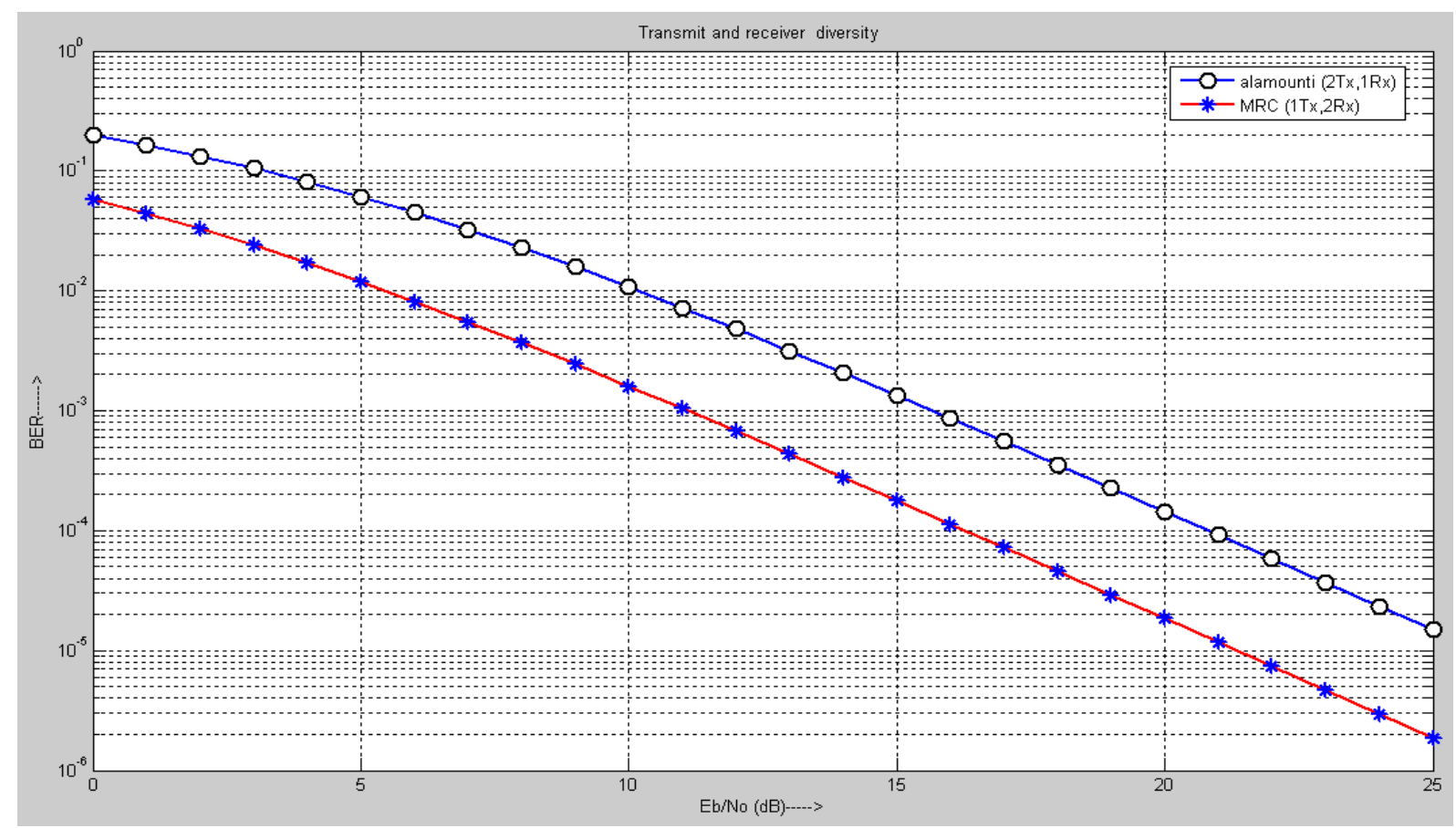

Figure 1: transmitter and receiver diversity with one user.

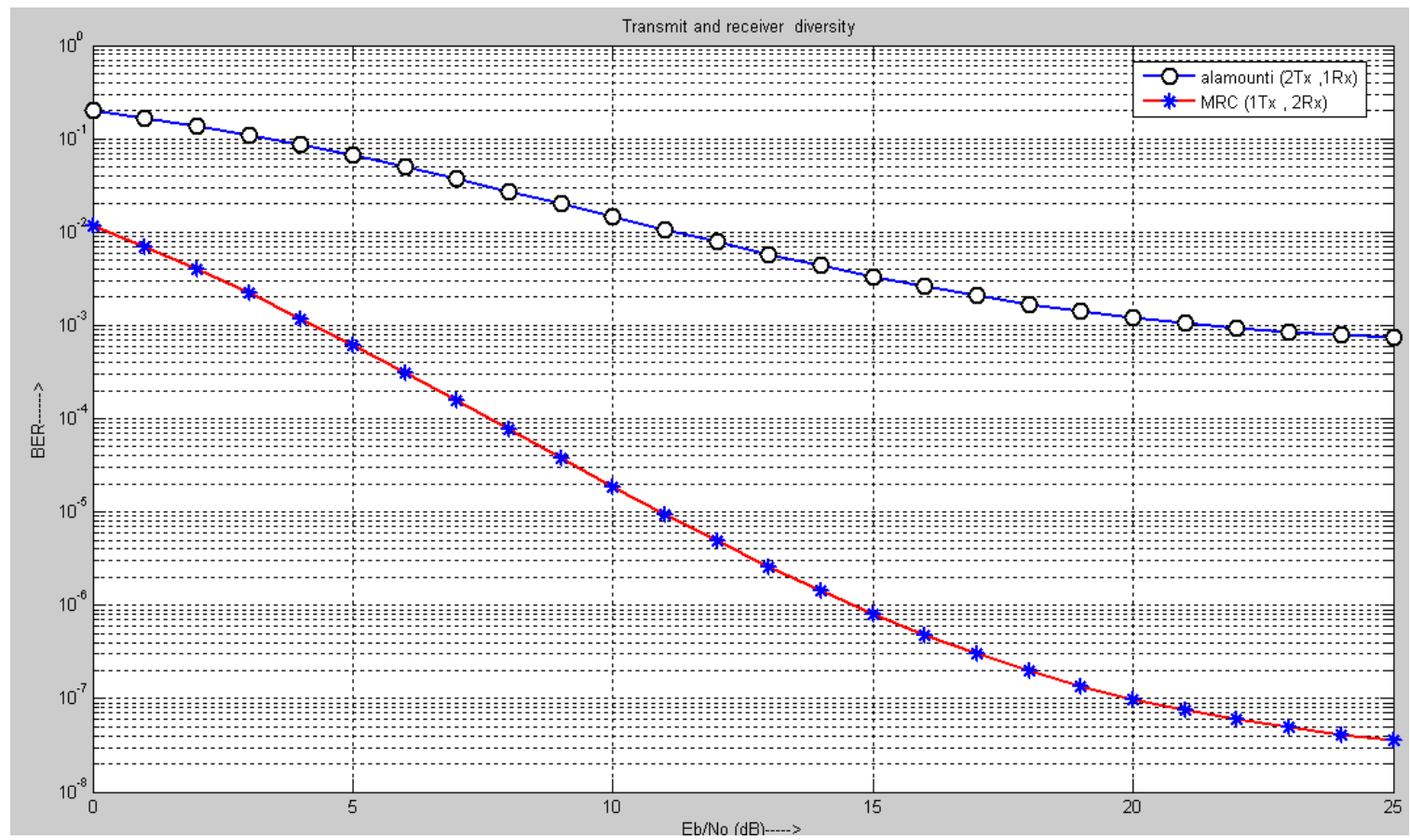

Figure 2: transmitter and receiver diversity with two user. 


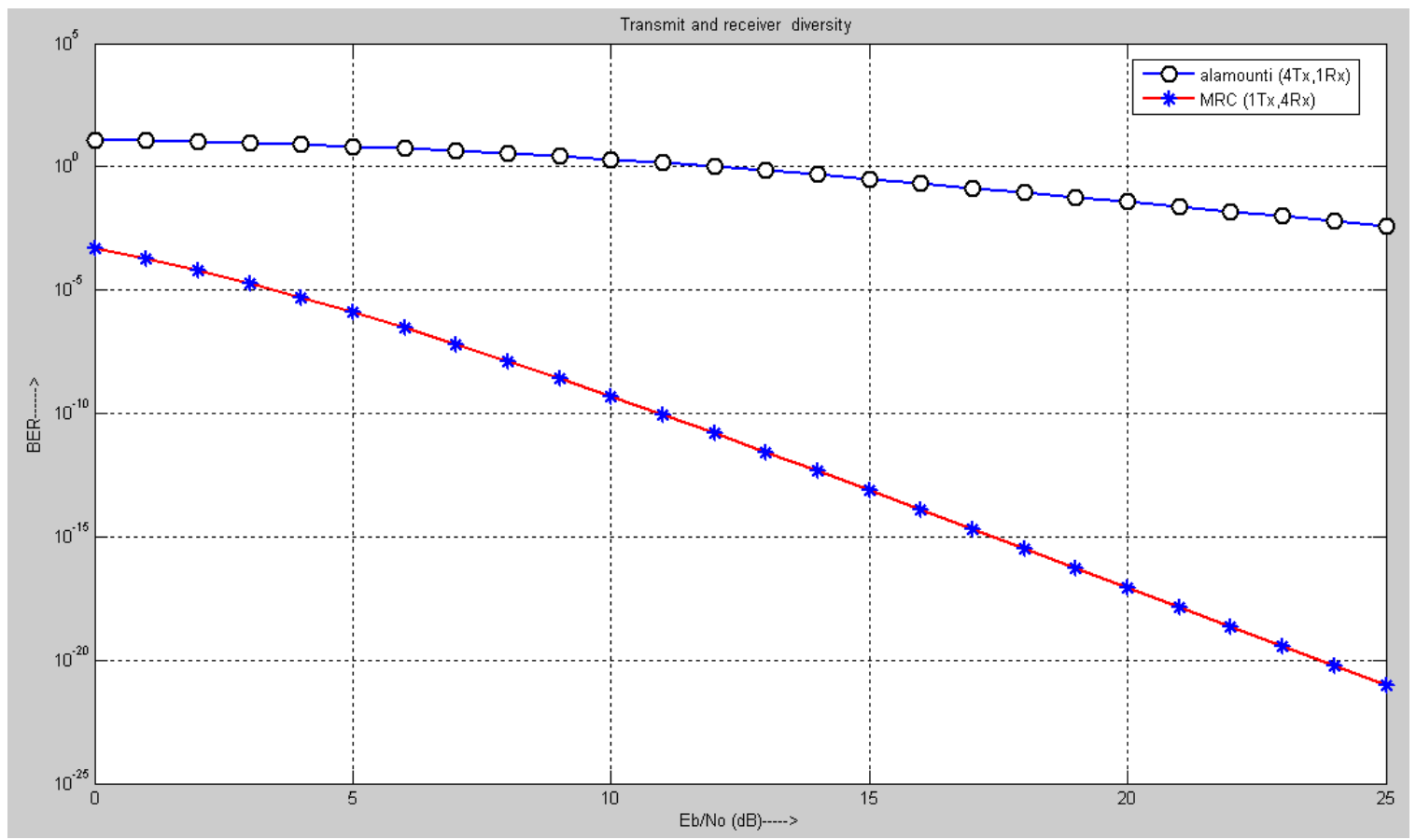

Figure 3: Transmitter and receiver diversity with one user and (4Tx, Rx and 1Tx, 4Rx).

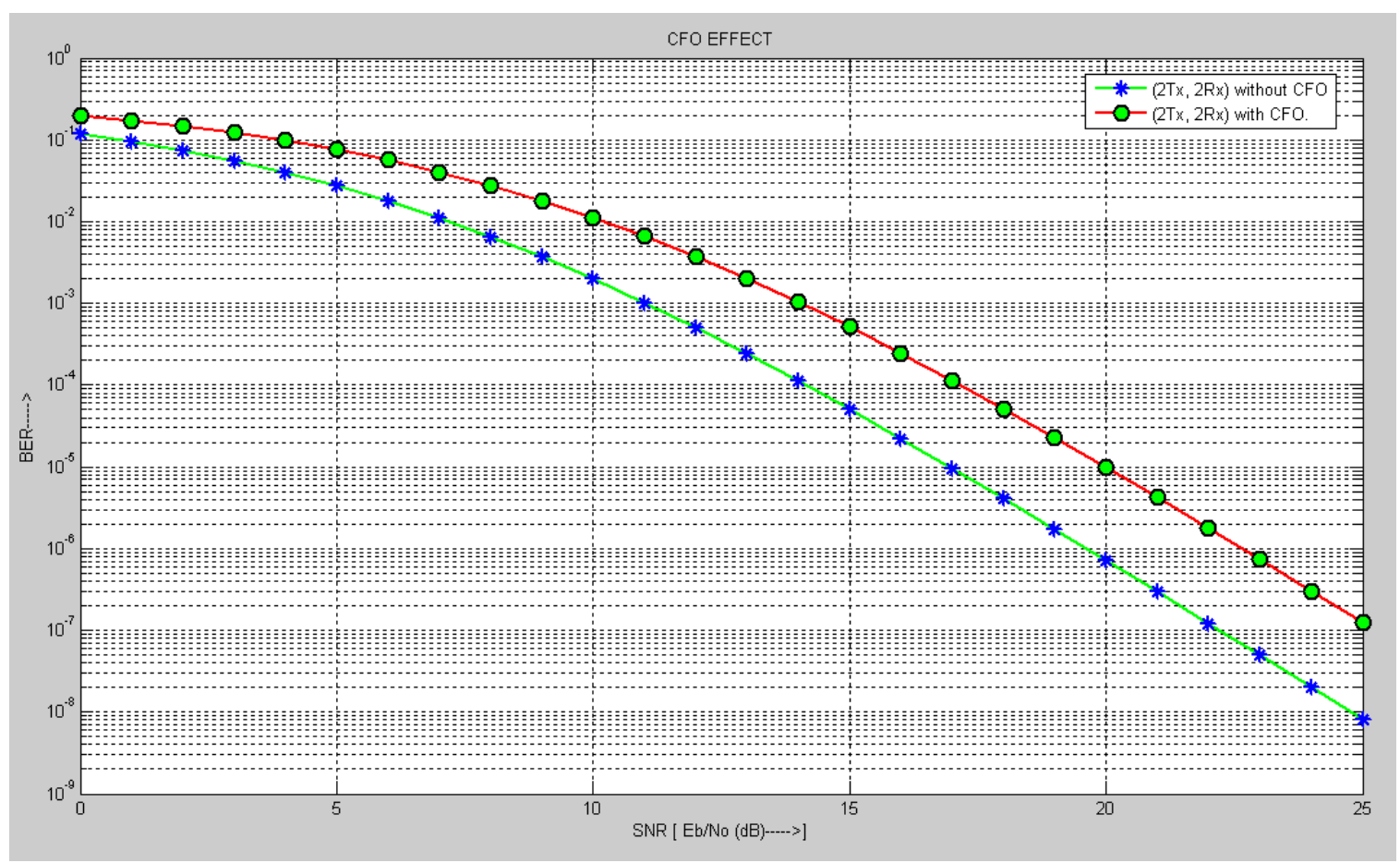

Figure 4: MIMO with CFO and without CFO (2 Tx and $2 \mathrm{Rx}$ for) one user. 


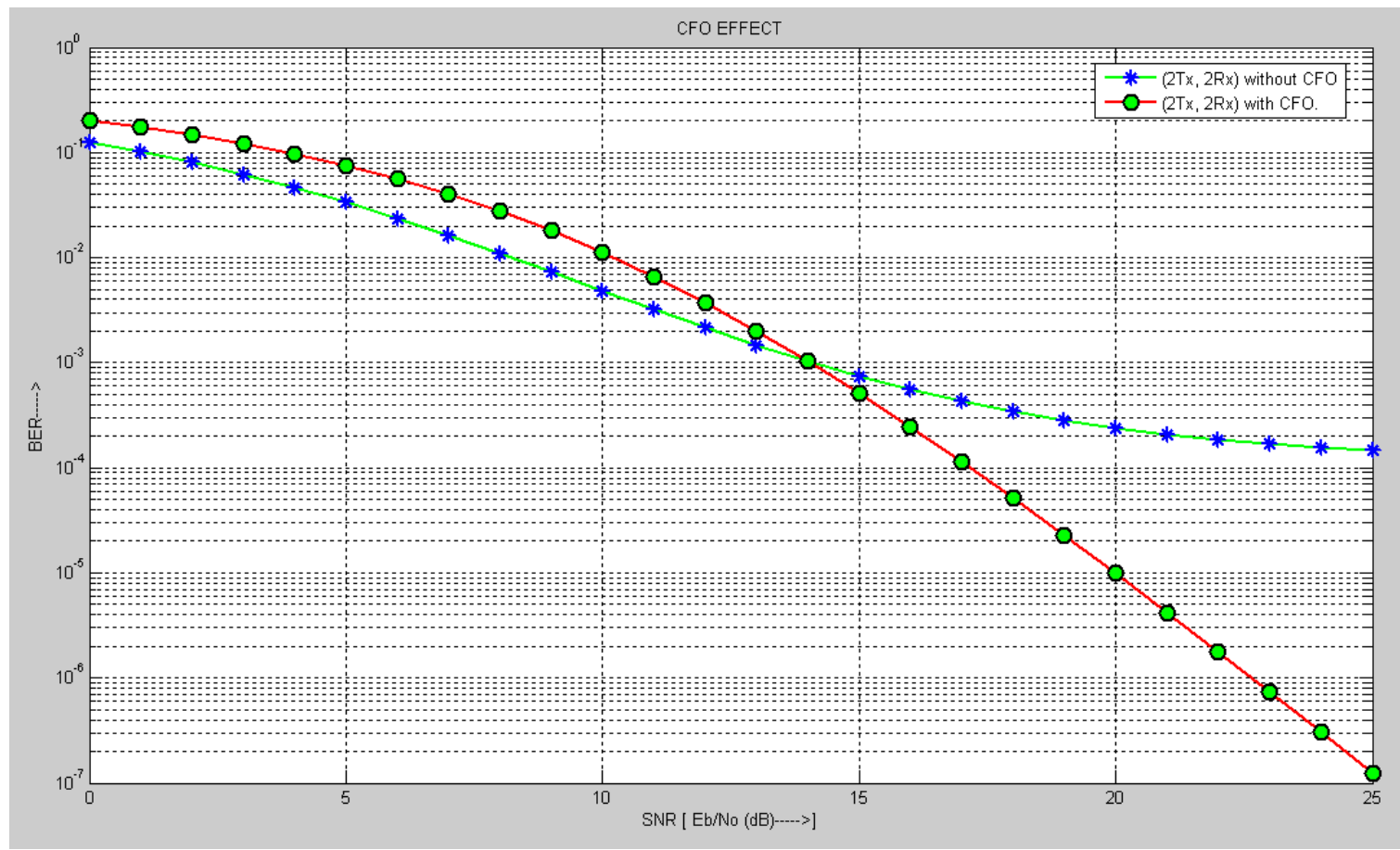

Figure 5: MIMO with CFO and without CFO (2Tx and 2Rx) for two user.

\section{Conclusion}

from the results and discussion above it was concluded that the receiver diversity is better than the transmitter diversity and when both diversity are applied the better results has been obtained. In addition to that MIMO without considering $\mathrm{CFO}$ in account is better for single user, but if there is more than one user at certain value of SNR become constant, thus the MIMO with CFO is not affected so it is better than MIMO without $\mathrm{CFO}$.

\section{REFERENCES}

[1] http://www.ece.clemson.edu.

[2] Vijaya Chandran Ramasam,, 2009,"BER performance over fading channels and diversity combining".

[3] Jyoteesh Malhotra ,2011,Performance Analysis of diversity Combining Multichannel Receivers in GenericGamma Fading Channels
[4] MD. ASIQUR RAHMAN and ABDUR RAHMAN, 2011, "Performance Analysis of a Space Time Block Coded MIMO System over Faded Channel". BRAC University, Dhaka, Bangladesh.

[5] Theodore.S.Rappaport,2002"Wireless Communications", Prentice-Hall Communications Engineering and Emerging Technologies Series.

[6] P. Barsocchi, ,2006, Channel models for terrestrial wireless communications: a survey, CNR-ISTI technical report.

[7] M. Awad, K. T. Wong and Z. Li, 2008, An Integrative Overview of the Open Literature's Empirical Data on the Indoor Radiowave Channel's Temporal Properties, IEEE Transactions on Antennas \& Propagation, vol. 56, no. 5, pp. $1451-1468$. 